\title{
The Impact of Non-compliance to Standardized Risk-adjusted Protocol in Non-Muscle Invasive Bladder Cancer on Recurrence, Progression and Mortality: Lessons to Learn in the Era of COVID-19
}

Faris Abushamma ( $\sim$ farisabushamma@hotmail.com )

An-najah National University Faculty of Medicine and Health Sciences https://orcid.org/0000-00020530-5466

\section{Zain Khayyat}

An-najah National University Faculty of Medicine and Health Sciences Aya Soroghle

An-najah National University Faculty of Medicine and Health Sciences

Sa'ed H. Zyoud

An-najah National University Faculty of Medicine and Health Sciences

Ahmad Jaradat

An-najah National University Faculty of Medicine and Health Sciences

Maha Akkawi

An-najah National University Faculty of Medicine and Health Sciences

\section{Hanood Aburass}

An-najah National University Faculty of Medicine and Health Sciences

lyad K. K. Qaddumi

An-najah National University Faculty of Medicine and Health Sciences

\section{Razan Odeh}

An-najah National University Faculty of Medicine and Health Sciences

Husam Salameh

An-najah National University Faculty of Medicine and Health Sciences

Salah Albuheissi

North Bristol NHS Trust

Research article

Keywords: NMIBC, COVID-19, Urological malignancies, haematuria

Posted Date: December 2nd, 2020 
DOl: https://doi.org/10.21203/rs.3.rs-116672/v1

License: (c) (1) This work is licensed under a Creative Commons Attribution 4.0 International License. Read Full License 


\section{Abstract}

\section{Background}

Non-Muscle Invasive Bladder Cancer (NMIBC) is potentially curable or a controllable disease if strict adherence to surveillance protocol is followed. Management and surveillance of NMIBC begin at the time of diagnosis up to few years thereafter based on the risk group category. There is scanty data in the literature evaluating the impact of non-compliance to surveillance protocol on progression, recurrence, and mortality rate. We do believe that this data will add to the literature especially during the COVID-19 pandemic which affects and delays daily elective surgeries.

\section{Methods}

A retrospective study of two hundred bladder biopsy and resection between 2012-2017. Eighty-eight patients with minimal 3 years follow up data were included. Patients were divided into 2 groups based on their compliance with the cystoscopy follow up as recommended by the European guidelines. We compared the cystoscopy compliant group to the non-compliant group in view of recurrence, progression, and mortality. In addition, missing variable items during surveillance are calculated in a new scoring model to predict the adverse outcome.

\section{Results}

Recurrence rate (RR), progression rate (PR), metastasis rate (MsR) and mortality rate (MR) are significantly higher in non-compliant group, RR: (92.6\%) ( $P<0.001)$, PR: (54.1\%) $(P<0.001)$, MsR: (37.7\%) $(P<0.001)$, MR: $(23.5 \%)(P=0.002)$ respectively. In subgroup analysis, intermediate and high-risk groups have a PR rate of zero in the compliant group while it is $100 \%(P<0.001)$ and $56.4 \%(P=0.001)$ in the noncompliant group respectively. Kaplan Meier (KM) graph shows that compliant patients were more likely to be better than non-compliant.

\section{Conclusions}

Noncompliance to a standardized surveillance protocol in NMIBC is associated statistically and clinically with adverse outcomes in comparison to a compliant group which mandates strict adherence to surveillance guidelines to improve outcomes in NMIBC.

\section{Background}

Bladder cancer is the seventh most common cancer in males and drops to be eleventh when both genders are considered [1]. In Europe, bladder cancer is the 5th most commonly diagnosed cancer and the 9th leading cause of mortality [2]. Nevertheless, bladder cancer is a broad category disease in view of surveillance and treatment. For example, non-muscle-invasive bladder cancer (NMIBC) represents the main category as $75 \%$ of patients newly diagnosed bladder cancer has a pathological stage of ( $\mathrm{Ta}$, Carcinoma in situ (CIS) and T1) [3]. The mainstay treatments of NMIBC are frequent trans-urethral 
resection of bladder tumor (TURBT), cystoscopies and Intravesical therapy such as intravesicalMitomycin (IV-MMC), and Bacillus Calmette-Guérin (BCG). European association of urology (EAU) strongly recommends following a risk stratification approach in NMIBC regarding cystoscopy surveillance and Intravesical therapy which mainly depends on tumor size, focality, grade, presence of CIS, and recurrences [4]. Several studies published the natural history of NMIBC mainly in view of recurrence and progression which led to creating a scoring model by EAU to predict them based on the number of tumors, tumor diameter, prior recurrence, concurrent CIS, and pathological stage and grade[ $[4,5]$.

There is strong evidence to support the importance of strict adherence to a surveillance protocol in NMIBC to decrease recurrence, progression, and improve cancer-specific survival [4]. However, the literature is sparse regarding the effect of non- adherence to a standardized protocol or the outcome of delaying surveillance cystoscopies. For instance, during the current COVID-19 pandemic, several papers and guidelines recommend delaying NMIBC treatment and surveillance especially in the low-risk category, Albeit, this is based on scanty data but no level one evidence with no consensus about the impact of such delay on tumour recurrence and progression [6].

Therefore, the goal of this study is to identify the impact of non-adherence to standardized risk-adjusted surveillance and intravesical treatment on recurrence rate (RR), progression rate (PR), and mortality rate (MR) based on our experience in the developing countries as we don not have a regional guidelines and not all centres follow the international guidelines. It is hoped that this research will contribute to a deeper understanding of the adverse outcomes associated with non-compliance to a surveillance protocol in NMIBC. Also, this may influence the decision of delaying treatment and surveillance of NMIBC in the era of the COVID-19 pandemic which may stay longer than previously expected [7].

\section{Methods}

\section{Study design}

It is a retrospective cohort study to evaluate the impact of non-compliance to standardized risk-adjusted surveillance and treatment in NMIBC. We have used the EAU risk stratification and surveillance protocol as a reference which is widely accepted and used in most European centres as we don't have national guidelines [8].

\section{Study Setting}

The data was collected from two tertiary urology centres dealing with bladder cancer in Northern Palestine which cover a total population of 300,000 .

\section{Study population and sampling}

We reviewed 20,000 histopathological reports in between 2012-2017. Two hundred bladder biopsies and resections reports were extracted 


\section{Inclusion criteria}

NMIBC confirmed pathology upon first or re-resection during the first 3 months of diagnosis and at least three years follow up

\section{Exclusion criteria}

Non-urothelial cell carcinoma pathology

\section{Data collection}

88 patients fit into either low, intermediate, or high-risk groups with at least 3 years follow up. We have classified them into 3 groups regarding compliance to surveillance cystoscopies:

\section{A. Group 0 (Compliant):}

The definition of compliance is not missing any cystoscopy in the low and intermediate group but missing none or maximum one in the high-risk group

\section{B. Group 1 (Non-compliant 1):}

Low and intermediate-risk: missing one or more cystoscopies.

High risk: missing two or more cystoscopies.

\section{Group 2 (Non -compliant 2)}

Low and intermediate: missing two or more cystoscopies.

High risk: missing three or more.

Each group was studied and compared to others in order to relate them with adverse outcomes (RR over 3 years, PR over 3 years (Progression defined as muscle invasion, prostatic stroma invasion, or development of regional lymphadenopathy on CT scan). In addition, the metastatic rate (MsR) and MR (Non-cancer-specific) also were evaluated. The clinical and statistical significance is noticed between group 0 and group 2, thus we focused our research on both groups.

\section{Statistical Analysis}

All analysis was performed with IBM Statistical Package for Social Sciences program (SPSS) Statistics version 21.0. Categorical variables were presented as absolute frequency (percentage). The Pearson's Chisquare test or Fisher's exact test, as appropriate, was applied to assess the differences in categorical variables. $P$ values $<0.05$ were considered statistically significant. Kaplan-Meier $(\mathrm{KM})$ techniques were used to generate mortality probabilities among adherent versus non-adherent patients in the follow-up of 
NMIBC with high-risk at each consecutive month until 5 years post-diagnosis. A scoring system is created to assess the effect of missing different variables during the surveillance of high-risk patients on RR, PR, and MR. The Scoring system composed of 4 items extracted from EAU surveillance protocol for the highrisk group as clarified in Table 1.

Table 1

Scoring System for high risk group.

\begin{tabular}{|ll|}
\hline Variables & Yes $=\mathbf{0}$ or No $=1$ \\
\hline (3 years follow up) & 0 : No missing or 1 \\
Number of missed cystoscopeis : & $1: 2$ missing \\
& $2: 3$ missing \\
IV-MMC & 0 or 1 \\
Re-resection at (4-6 weeks) & 0 or 1 \\
\hline BCG( Induction and at least M1) & 0 or 1 \\
\hline Total = & $<3$ or $\geq 3$ \\
\hline
\end{tabular}

\section{Results}

\section{Demographic and clinical presentation}

The mean age of diagnosis is 60 years old ( $M=60, S D: 3.45)$. The male gender is the predominate as 78 (88.6\%) were males. Most of the patients are smokers $(73,83 \%)$ but with no family history of bladder cancer as positive family history were noticed just in 17 (19\%) patients. Significant medical comorbidities were found in more than half of the patients where hypertension (HTN) represents the most common and diabetes mellitus (DM) represents the second most common. The most common presentation was haematuria ( $\mathrm{VH}$ and $\mathrm{NVH}) 63(71.6 \%)$. Half of the patients had the diagnosis made after 2 weeks (M: 8.3, SD:19.2);(Table 2A).

\section{Staging, grading and risk stratification}

66 (75\%) had a lack of accurate pathological staging but vaguely described as papillary NMIBC. However, grading was accurately recorded where the majority $(66,75 \%)$ of the specimens were high grade (G2-3). $47 \%$ had muscle during the first resection, Nevertheless, the rest of our cohort had confirmatory NMIBC diagnosis during re-resection during the first 3 months of diagnosis.

The patients were distributed into three main risk group categories according to the EAU risk stratification [8]. The largest group was the high-risk group which contains 63 patients $(71.5 \%)$, while the low and intermediate group entails 25 patients (28.5\%); (Table 2B). 


\section{General compliance to surveillance cystoscopy, RR, PR, MsR, and MR.}

Generally, most of the cohort are not compliant to the surveillance cystoscopy protocol as just 20 (23\%) patients were compliant. More than $80 \%$ had recurrence tumors over the 3 years follow up. Progression was noticed in $34(38.6 \%)$ but 8 (9\%) had no data available about progression. The MR is around $18 \%$ (Table 2C). 
Table 2

Sociodemographic and clinical characteristics of participants $(n=88)$

\section{Variables}

Number of participants (\%)

Table 2-A demographic and clinical presentation

\section{Age}

$<60$

23(26.1)

$\geq 60$

65(73.9)

Gender

Male

78(88.6)

Female

10(11.4)

\section{Smoking}

Yes

No

15(17.0)

\section{Family history}

Yes

17(19.3)

No

71(80.7)

Co-morbidities (HTN , DM or IHD)

Yes

65(73.9)

No

23(26.1)

\section{Presentation of disease}

Hematuria

Scans (Ultrasound , CT scan , .. etc )

LUTS (mainly dysuria )

Others

$4(4.5)$

Table 2-B: Risk stratification staging and grading

\section{Stage of tumor}

$\mathrm{Pa}$

12(13.6)

Pt1 


\section{Grade of tumor}

Low/PUNLMP

22(25.0)

High

$66(75.0)$

\section{Risk group}

Low

14(15.9)

Intermediate

High

63(71.6)

\section{Delay of diagnosis}

Yes

44(50.0)

No

$44(50.0)$

\section{Muscle found in first resection}

Yes

41(46.6)

No

$47(53.4)$

IV-MMC given at $1^{\text {st }}$ resection

Yes

No

$79(89.8)$

Table 2-C: Compliance, RR, PR, MsR and MR Follow up over 3 years

\section{Compliance for all groups}

Yes

20(22.7)

No

68(77.3)

Compliance Rate for each risk

Low

Intermediate

High

\section{Recurrence}

Yes

No

14(15.9) 


\section{Progression}

Yes

34(38.6)

No

46(52.3)

Missing

8(9.1)

\section{Metastasis}

Yes

No

Missing

8(9.1)

Mortality Rate

16(18.2)

LUTs: lower urinary tract symptoms; CT: computed temoghraphy scan; BCG: Bacillus CalmetteGuérin; IV-MMC: intra-vesical Mitomycin; DM: diabetes mellitus; HTN: hypertension; IHD: Ischemic heart disease; RR: Recurrence Rate; PR: Progression Rate; MsR: Metastatic Rate; MR: mortality Rate.

\section{Compliant versus non- compliant}

RR, PR, MsR and MR are significantly higher in non-compliant group, RR: (92.6\%) $(P<0.001), \mathrm{PR}$ : (54.1\%) $(P<0.001)$, MsR: $(37.7 \%)(P<0.001)$, MR: $(23.5 \%)(P=0.002)$ respectively. Obviously, patients older than 60 years old is less compliant to surveillance cystoscopy protocol 56 (82\%) $(P=0.001)$; (Table 3). 
Table 3

Sociodemographic and clinical aspects in correlation to compliance.

\begin{tabular}{|c|c|c|c|c|}
\hline Variables & $\begin{array}{l}\text { Compliant } \\
\mathrm{N}=20 \text { (\%) }\end{array}$ & $\begin{array}{l}\text { Non-compliant } \\
\mathrm{N}=68\end{array}$ & Total & $P$ value ${ }^{a}$ \\
\hline $\begin{array}{l}\text { Gender } \\
\text { Male } \\
\text { Female }\end{array}$ & $\begin{array}{l}19(95 \%) \\
1(5 \%)\end{array}$ & $\begin{array}{l}59(86.8) \\
9(13.2)\end{array}$ & $\begin{array}{l}78(88.6 \%) \\
10(11.4 \%)\end{array}$ & $0.270^{b}$ \\
\hline $\begin{array}{l}\text { Age } \\
<60 \\
>60\end{array}$ & $\begin{array}{l}11(55 \%) \\
9(45 \%)\end{array}$ & $\begin{array}{l}12(17.6) \\
56(82 \%)\end{array}$ & $\begin{array}{l}23(26.1 \%) \\
65(73.9 \%)\end{array}$ & $0.001^{c}$ \\
\hline $\begin{array}{l}\text { Smoking } \\
\text { Yes } \\
\text { No }\end{array}$ & $18(90.0 \%)$ & 55 (80.9\%) & $73(83.0 \%)$ & $0.317^{b}$ \\
\hline $\begin{array}{l}\text { Family history } \\
\text { Yes } \\
\text { No }\end{array}$ & $\begin{array}{l}20(100.0 \%) \\
0(0.0 \%)\end{array}$ & $\begin{array}{l}52(76.5 \%) \\
16(23.5 \%)\end{array}$ & $\begin{array}{l}72(81.8 \%) \\
16(18.2 \%)\end{array}$ & $0.002^{b}$ \\
\hline Co-morbidities & $12(60.0 \%)$ & $53(77.9 \%)$ & 65 (73.9\%) & $0.108^{c}$ \\
\hline $\begin{array}{l}\text { Delay of diagnosis } \\
\text { (Within } 2 \text { weeks of presentation) }\end{array}$ & $\begin{array}{l}16(80.0 \%) \\
4(20.0 \%)\end{array}$ & $\begin{array}{l}47(69.1 \%) \\
21(30.9 \%)\end{array}$ & $\begin{array}{l}63(71.6 \%) \\
25(28.4 \%)\end{array}$ & $0.002^{c}$ \\
\hline $\begin{array}{l}\text { Recurrence } \\
\text { Yes } \\
\text { No }\end{array}$ & $\begin{array}{l}11(55.0 \%) \\
9(45.0 \%)\end{array}$ & $\begin{array}{l}63(92.6 \%) \\
5(7.4 \%)\end{array}$ & $\begin{array}{l}74(84.1) \\
14(15.9)\end{array}$ & $<0.001^{c}$ \\
\hline $\begin{array}{l}\text { Progression } \\
\text { Yes } \\
\text { No }\end{array}$ & $\begin{array}{l}1(5.3 \%) \\
18(94.7 \%)\end{array}$ & $\begin{array}{l}33(54.1 \%) \\
28(45.9 \%)\end{array}$ & $\begin{array}{l}34(42.5) \\
46(57.5)\end{array}$ & $<0.001^{b}$ \\
\hline
\end{tabular}

${ }^{a} P$ values in bold are below the significance level of 0.05 .

b Statistical significance values calculated using Fisher's Exact Test.

c Statistical significance values calculated using the Pearson Chi-Square. 


\begin{tabular}{|c|c|c|c|c|}
\hline Variables & $\begin{array}{l}\text { Compliant } \\
\mathrm{N}=20(\%)\end{array}$ & $\begin{array}{l}\text { Non-compliant } \\
\mathrm{N}=68\end{array}$ & Total & P value ${ }^{a}$ \\
\hline Mets & $1(5.3 \%)$ & $23(37.7 \%)$ & $24(30.0 \%)$ & $0.003^{b}$ \\
\hline Yes & $18(94.7 \%)$ & $38(62.3 \%)$ & $56(70.0 \%)$ & \\
\hline \multicolumn{5}{|l|}{ No } \\
\hline Mortality Rate & $0(0.0 \%)$ & $16(23.5 \%)$ & $16(18.2 \%)$ & $0.002^{b}$ \\
\hline \multicolumn{5}{|c|}{ a $P$ values in bold are below the significance level of 0.05 . } \\
\hline \multicolumn{5}{|c|}{ b Statistical significance values calculated using Fisher's Exact Test. } \\
\hline \multicolumn{5}{|c|}{ c Statistical significance values calculated using the Pearson Chi-Square. } \\
\hline
\end{tabular}

The major statistical significant is seen when you compare Group 0 (compliant) to group 2 (Noncompliant 2) as for instance, in the intermediate rate and high-risk group, the PR rate in the compliant group is none while it is $100 \%(P<0.001)$ and $56.4 \%(P=0.001)$ in the non-compliant group respectively (Table 4). Furthermore, the MR is statically significantly higher in the high-risk group $25 \%(P=0.03)$ which mandates us to evaluate the mortality rate over 5 years using $\mathrm{KM}$ curve.

Table 4

The correlation between compliance in each risk group and RR, PR and MR.

\begin{tabular}{|c|c|c|c|c|c|c|c|c|c|}
\hline \multirow{2}{*}{$\begin{array}{l}\text { Risk } \\
\text { Compliance }\end{array}$} & \multicolumn{3}{|c|}{ Low risk } & \multicolumn{3}{|c|}{ Intermediate risk } & \multicolumn{3}{|c|}{ High risk } \\
\hline & $\mathrm{RR}(\%)$ & $\operatorname{PR}(\%)$ & $\operatorname{MR}(\%)$ & $\mathrm{RR}(\%)$ & PR(\%) & $\mathrm{MR}(\%)$ & $\mathrm{RR}(\%)$ & PR(\%) & $\mathrm{MR}(\%)$ \\
\hline Compliant & 33.3 & 16.7 & 0.0 & 40.0 & 0.0 & 0.0 & 77.8 & 0.0 & 0.0 \\
\hline $\begin{array}{l}\text { Non- } \\
\text { compliant } 2\end{array}$ & 100.0 & 83.3 & 28.6 & 100.0 & 100.0 & 16.7 & 93.2 & 56.4 & 25.0 \\
\hline P value ${ }^{a}$ & $.004^{b}$ & $.016^{\mathrm{b}}$ & .095 & $.013^{b}$ & $\begin{array}{l}< \\
0.001 \\
b\end{array}$ & .255 & .195 & $.001^{\mathrm{b}}$ & $.03^{b}$ \\
\hline \multicolumn{10}{|c|}{ RR: Relative Risk, PR: Progression Risk, MR: Mortality Rate } \\
\hline \multicolumn{10}{|c|}{ a $P$ values in bold are below the significance level of 0.05 . } \\
\hline
\end{tabular}


Forty-five patients were diagnosed with NMIBC between January 2012 and May 2015. Among these patients, 7 were compliant and 38 were non-compliant. We followed them up until May 2020. 15 deaths were found and all of them were non-compliant. To conclude, KM graph shows that compliant patients were more likely to be better than non-compliant. This finding did not reach statistical significance $(P=$ 0.075) because all died patients were non-compliant (Fig. 1).

\section{Scoring system to predict recurrence, progression, and mortality in high-risk patients with poor compliance}

A scoring system has been created to assess the effect of missing different variables during the surveillance of high-risk patients on RR, PR, and MR. Thirty-six patients from the high-risk group were assessed regarding the compliance to EAU high-risk surveillance protocol mainly procedures and treatments which is proven to have an impact of recurrence, progression, and mortality. Therefore, we assess the statistically significant of having a score three or more in relation to RR, PR and MR. Scoring three or more is statistically and clinically significant associated with higher recurrence, progression, and mortality. RR: (94\%) $(P=0.016)$, PR: $49 \%(P<0.001)$ and MR $(26 \%)(P=0.012)$; (Table 5).

Table 5

Novel Scoring System to predict recurrence, progressionand mortality in high risk group.

\begin{tabular}{|llll|}
\hline Outcome & $\begin{array}{l}\text { Number of }<3 \\
(\%)\end{array}$ & $\begin{array}{l}\text { Number of } \geq 3 \\
(\%)\end{array}$ & P value $^{\mathrm{a}}$ \\
\hline $\begin{array}{l}\text { Recurrence } \\
\text { Yes }\end{array}$ & $8(67.0)$ & $48(94.1)$ & $\mathbf{0 . 0 1 6}^{\mathrm{b}}$ \\
\hline $\begin{array}{l}\text { Progression } \\
\text { Yes }\end{array}$ & $0(0.0)$ & $22(48.9)$ & $<\mathbf{0 . 0 0 0 1}^{\text {b }}$ \\
\hline $\begin{array}{l}\text { Mortality } \\
\text { Yes }\end{array}$ & $12(25.5)$ & $\mathbf{0 . 0 1 2}^{\mathrm{b}}$ \\
\hline $\begin{array}{l}\text { a } P \text { values in bold are below the significance level of 0.05. } \\
\text { b Statistical significance values calculated using Fisher's Exact Test. }\end{array}$ & \\
\hline
\end{tabular}

\section{Discussion}

Our study has been performed during this period of a worldwide COVID-19 pandemic negatively affecting the health system and led to a significant burden on hospitals and daycare units[7]. We have evaluated the outcomes of non - compliance to surveillance protocol in the management of NMIBC to further understand the natural history of this disease and outcomes of delaying surveillance cystoscopies as it is currently suggested by several published articles[6]. Obviously, our data shows that the PR and RR are 
significantly higher in the non-compliant group which highlights the importance of following a validated surveillance protocol and not delaying surveillance cystoscopies or missing intravesical treatment despite the current challenges facing the health system globally during the COVID-19 pandemic mainly in the high-risk group.

NMIBC diagnosis and management algorithm is well established where various international protocols and guidelines are available. For instance, EAU annually updates the guidance on NMIBC surveillance and management, in addition to creating a scoring model to predict progression and recurrence which are the most important factors in NMIBC prognosis [8].

However, in Palestine and other regions in the Middle East, there is no local guidance to manage NMIBC which makes expert opinion and international guidelines are both the mainstay way of diagnosis and management. Furthermore, patient compliance is an issue for several reasons such as financial problems, false beliefs, and more recently the current COVID-19 pandemic. Therefore, we have used this deviation from the standard of care to answer the compelling question in the literature about how strict we should be following surveillance cystoscopies and other treatments in NMIBC management.

Few articles recently raised a question regarding the impact of overuse cystoscopy in low-risk NMIBC on cost and hospital occupancy, on the contrary, underuse in high risk may be associated with serious adverse outcomes. Therefore, a huge debate has been raised recently regarding the strict adherence to surveillance cystoscopies taking into account that NMIBC disease is a wide spectrum disease ranging from low risk to high risk with no available current data regarding the impact of noncompliance to a standardized risk-adjusted protocol on recurrence, progression and mortality $[9,10]$. Furthermore, several papers currently have been published to safely organize urology work during the COVID-19 pandemic which includes the suggestion of delaying NMIBC surveillance and treatment [6]. This evolving concept may harbor hidden harm if not well studied mainly in the high-risk group which is the group most commonly associated with recurrence and progression according to the EAU scoring prediction model and also it is the most prevalent in our study $(71.5 \%)[8,9]$. Furthermore, there is no available data to look comprehensively into the effect of non-compliance to the bladder cancer diagnosis and management pathway especially in the high-risk group which also includes the time of diagnosis, imaging, cytology, and intravesical therapy. Our data shows that the non-compliant NMIBC group has got higher RR, PR and MR. This outcome supports the importance of adherence to surveillance protocols regardless to the stage but more importantly in the high-risk group as our data shows that the PR rate in the compliant group is none while it is $100 \%(P \leq .001)$ in non-compliant intermediate group and $56.4 \%(P=.001)$ in noncompliant high-risk group. Thus, NMIBC management especially high-risk category should be prioritized during the current COVID-19 pandemic and should not be delayed.

National Institute for health and care excellence guidelines (NICE guidelines) recommend a two-week wait pathway to diagnose bladder cancer especially if haematuria is the main presentation [10]. Our results showed that half of the patients had a delay in diagnosis despite having haematuria as the most prevalent presentation in our cohort which may also contribute to the overall bad prognosis. Furthermore, 
at the time of the first resection $90 \%$ failed to have IV-MMC which may explain the high RR in all subgroups as single, immediate post-operative IV-MMC significantly reduces the RR compared to TURBT alone [11]. It is obvious that missing more items during NMIBC management is associated with the poorer outcome as our scoring model shows that scoring three or more is significantly associated with higher recurrence, progression and mortality. This can be used in regions where compliance to surveillance protocol is an issue to counsel patients about the negative impact on poor compliance on recurrence, progression and mortality. KM curve shows that compliant patients were more likely to do better than the non-compliant group over 5 years follow up

The high-risk group carries the highest risk of progression. Therefore, this category has been thoroughly evaluated in order to decrease the risk of progression which affects overall prognosis. Several strategies have been recommended to treat this group such as full-dose intravesical BCG for one to three years, creation a subgroup of highest risk tumours by EAU and upfront cystectomy in some cases $[4,12,13]$. In our article, the group 2 non-compliant high-risk group is associated with a significant PR and quarter MR. Thus, adherence to a surveillance protocol in the high-risk group is strongly recommended and should be followed strictly. More importantly, this will help to answer the trending question in the literature regarding the importance of surveillance cystoscopies during the COVID-19 pandemic which negatively affects health economic, medical personal, and hospital occupancy [14].

\section{Limitations Of The Study}

This study has got few limitations such as a small sample size, few patients lost to follow up, or had some data not available, and lack of standardization of pathology reports. This might have led to missing the primary diagnosis of NMIBC initially in a few cases which may explain the higher MsR and MR. However, this study represents the outcome of deviation from the standard of care and help to clarify the impact of it on prognosis and associated adverse outcomes especially in the high-risk group.

\section{Conclusions}

NMIBC is a potentially controllable disease with an excellent outcome if surveillance protocol is applied and followed. However, this study shows that the noncompliance group has a significantly worse outcome in comparison to the compliant group. RR, PR, and MR are higher in the non-compliance group. Thus, strict adherence to international guidelines is strongly recommended especially in the high-risk group and NMIBC management should be prioritized in the era of the COVID-19 pandemic.

\section{List Of Abbreviations}

NMIBC, Non-muscle Invasive Bladder Cancer; CIS, Carcinoma in situ; TURBT, Trans-urethral Resection of Bladder Tumour; IV-MMC, Intravesical Mitomycin; BCG, Bacillus Calmette-Guérin, EAU; European association of urology; RR, Recurrence Rate; PR, Progression Rate; MR, Mortality Rate; MsR, Metastatic 
Rate; SPSS, Statistical Package for Social Sciences program; KM, Kaplan Miere; DM, diabetes mellitus; NICE, National Institute for Health and Care excellence.

\section{Declarations}

\section{Ethical approval and consent to participate}

This study has been approved by the Institutional Review Board of An-Najah National University, and the approval from the Palestinian Ministry of Health. The IRB approval can be provided if required. (Archived number 7 Jan 2020).

\section{Consent for publication}

Not applicable.

\section{Availability of data and materials}

All datasets collected and analysed in this survey will be available by the corresponding author upon any reasonable request.

\section{Competing interests}

All authors declare there are no competing interests.

\section{Funding}

No specific grant was received from any public, commercial or non-profit funding agency for this research.

\section{Acknowledgement}

Many thanks to Clinical Research Centre of An-Najah National University Hospital for constant support and wise advices.

\section{Author contributions:}

FA, ZK and AS collected and analysed the data, performed literature review and contributed to manuscript drafting. FA and SZ conducted data analysis and literature review and partially interpreted the data and 
drafted the manuscript. IQ, AJ, RO and HS participated in the conception and design of the study and data interpretation. HA, MA and SA played a key role in conceptualization, designing, coordination, supervision of this study and took the responsibility of the integrity of the data and the accuracy of the analysis; he also conducted a critical review of results' interpretation and took part in the final writing. All authors read the final manuscript and approve of it.

\section{References}

1. Ferlay J, Soerjomataram I, Dikshit R, Eser S, Mathers C, Rebelo M, Parkin DM, Forman D, Bray F: Cancer incidence and mortality worldwide: sources, methods and major patterns in GLOBOCAN 2012. Int J Cancer 2015, 136(5):E359-386.

2. Larsson SC, Andersson SO, Johansson JE, Wolk A: Diabetes mellitus, body size and bladder cancer risk in a prospective study of Swedish men. Eur J Cancer 2008, 44(17):2655-2660.

3. Compérat E, Larré S, Roupret M, Neuzillet Y, Pignot G, Quintens H, Houéde N, Roy C, Durand X, Varinot $\mathrm{J}$ et al: Clinicopathological characteristics of urothelial bladder cancer in patients less than $\mathbf{4 0}$ years old. Virchows Arch 2015, 466(5):589-594.

4. Babjuk M, Böhle A, Burger M, Capoun O, Cohen D, Compérat EM, Hernández V, Kaasinen E, Palou J, Rouprêt $\mathrm{M}$ et al: EAU Guidelines on Non-Muscle-invasive Urothelial Carcinoma of the Bladder: Update 2016. Eur Urol 2017, 71(3):447-461.

5. Sylvester RJ, van der Meijden AP, Oosterlinck W, Witjes JA, Bouffioux C, Denis L, Newling DW, Kurth K: Predicting recurrence and progression in individual patients with stage Ta T1 bladder cancer using EORTC risk tables: a combined analysis of 2596 patients from seven EORTC trials. Eur Urol 2006, 49(3):466-465; discussion 475-467.

6. Abushamma F, Jaradat A, Al-azab R, Demour SA, Albuheissi S, Mustafa A, Alzarooni A, Alansari A, AlRumaihi K, Aboumarzouk OM: Recommendations and algorithm to deal with urological surgeries in the Middle East during COVID-19 Pandemic: Review article and regional expert opinion.Pal Med Pharm J 2020, 5(2):133-139.

7. Huang C, Wang Y, Li X, Ren L, Zhao J, Hu Y, Zhang L, Fan G, Xu J, Gu X et al: Clinical features of patients infected with 2019 novel coronavirus in Wuhan, China. Lancet 2020, 395(10223):497-506.

8. Taylor J, Becher E, Steinberg GD: Update on the guideline of guidelines: non-muscle-invasive bladder cancer. BJU Int 2020, 125(2):197-205.

9. Datovo JCF, Neto WA, Mendonca GB, Andrade DL, Reis LO: Prognostic impact of non-adherence to follow-up cystoscopy in non-muscle-invasive bladder cancer (NMIBC). World J Urol 2019, 37(10):2067-2071.

10. National Institute for Health and Care Excellence (NICE). Suspected cancer: recognition and referral; NICE guideline [NG12]. 2015. https://www.nice.org.uk/guidance/ng12/chapter/1-Recommendationsorganised-by-site-of-cancer\#urological-cancers (accessed June 13 2020). 
11. Sylvester RJ, Oosterlinck W, Holmang S, Sydes MR, Birtle A, Gudjonsson S, De Nunzio C, Okamura K, Kaasinen E, Solsona E et al: Systematic Review and Individual Patient Data Meta-analysis of Randomized Trials Comparing a Single Immediate Instillation of Chemotherapy After Transurethral Resection with Transurethral Resection Alone in Patients with Stage pTa-pT1 Urothelial Carcinoma of the Bladder. Which Patients Benefit from the Instillation?Eur Uro/ 2016, 69(2):231-244.

12. Sylvester RJ, van der MA, Lamm DL: Intravesical bacillus Calmette-Guerin reduces the risk of progression in patients with superficial bladder cancer: a meta-analysis of the published results of randomized clinical trials. J Uro/ 2002, 168(5):1964-1970.

13. Faba OR, Palou J, Breda A, Villavicencio H: High-risk non-muscle-invasive bladder cancer: update for a better identification and treatment. World J Uro/2012, 30(6):833-840.

14. Mostafid H: Cystoscopic surveillance for bladder cancer: Learning the lessons forced upon us by the Covid-19 pandemic. Scand J Urol 2020:DOI: 10.1080/21681805.21682020.21794956.

\section{Figures}

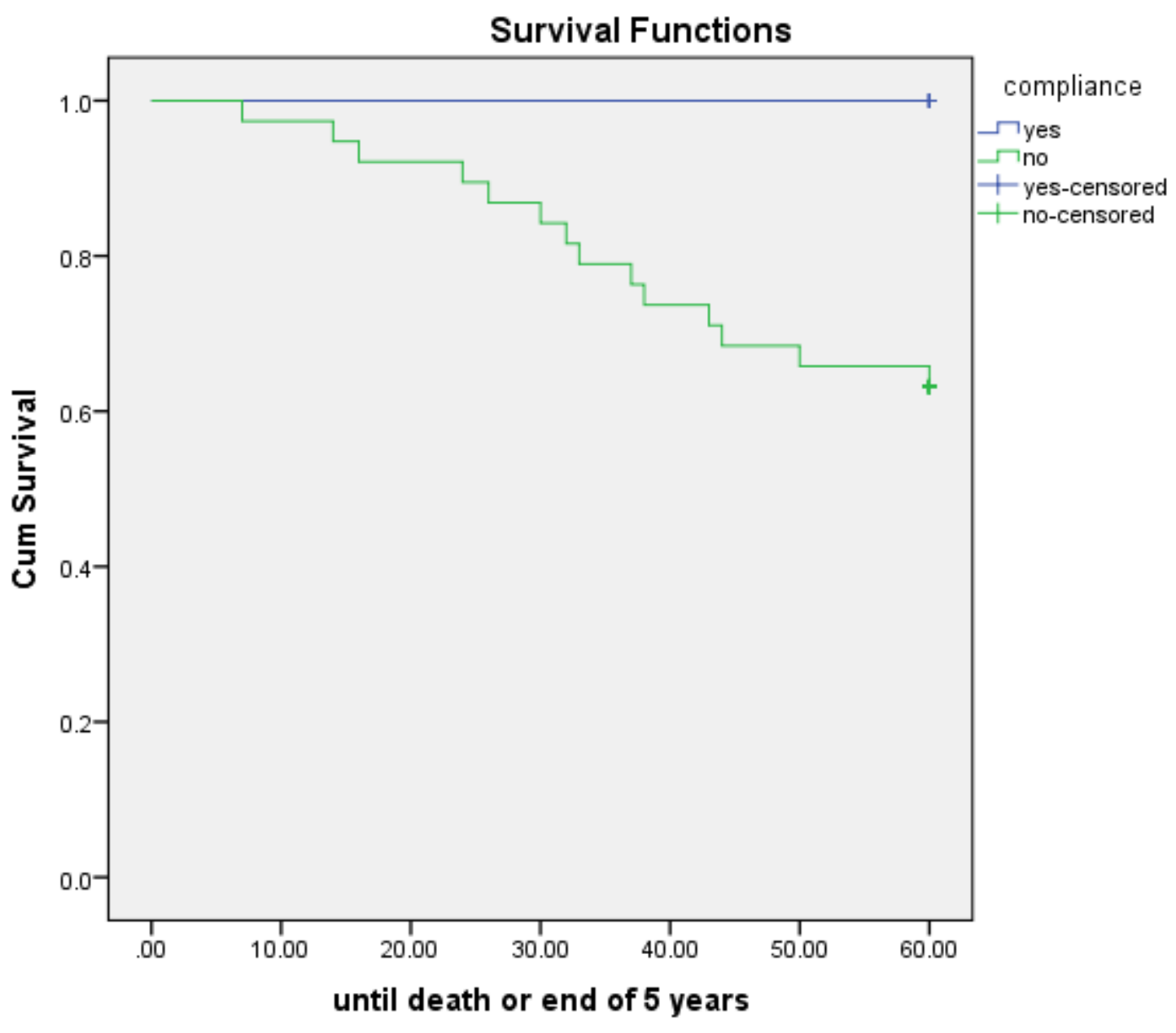

Figure 1 
Comparison of the estimated probability of mortality among compliant versus non- compliant patients in the follow-up of NMIBC with high-risk based on the Kaplan-Meier method for 5 years post-diagnosis

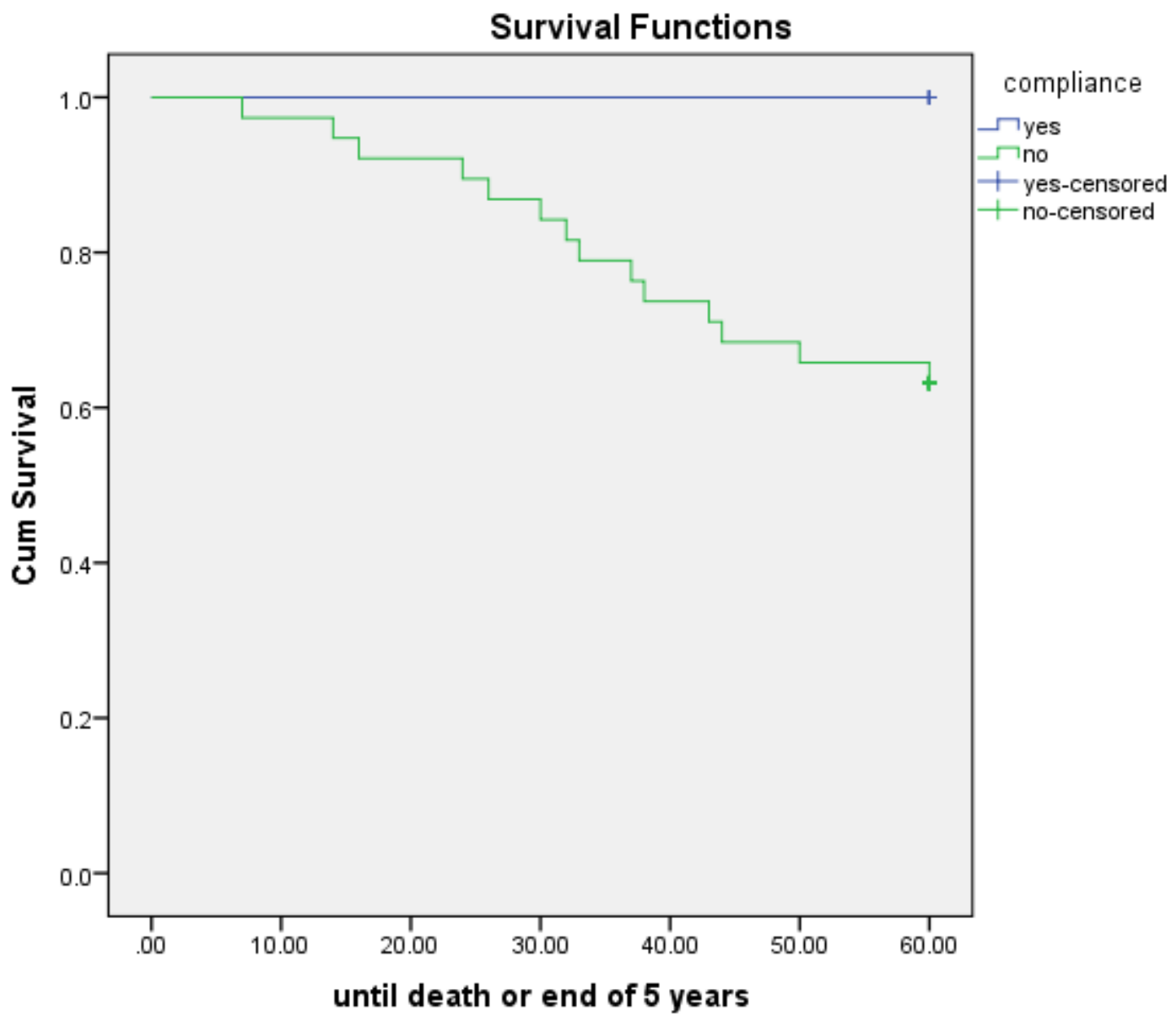

Figure 1

Comparison of the estimated probability of mortality among compliant versus non- compliant patients in the follow-up of NMIBC with high-risk based on the Kaplan-Meier method for 5 years post-diagnosis 